\title{
APPLYING QUANTITATIVE TECHNIQUES TO EVALUATE THE LEVEL OF SUSTAINABILITY FOR PHYSICAL DIMENSION OF URBAN FORM IN SANANDAJ CITY, IRAN
}

\author{
FARZIN CHAREHJOO \& HO CHIN SIONG \\ Faculty of Built Environment, Department of Urban and Regional Planning, \\ Universiti Teknologi Malaysia, Kuala Lumpur, Malaysia.
}

\begin{abstract}
This paper aims to understand and evaluate the sustainability of physical dimension of urban form in rapidly growing urban areas and presents some quantitative methods (Shannon's Entropy and Holden Apportioning Technique) to measure the level of sustainability for urban form as a practical implication. The rapid urbanization in Iran in the second half of the 20th century has mostly manifested itself in the emergence of large cities and issue of sustainable urban form is becoming ever more important, taking into consideration the contemporary urban population growth and the concern of sustainable development. The Sanandaj City is located on the western side of Iran, and has a population of about 400,000. There has been rapid urbanization during the last three decades in the city and it is therefore chosen as a case study. The results of these measurements indicate that in order to achieve sustainable urban form, the urban policies for development should be changed. A more rational and focused approach is required in development of the urban areas to achieve efficacy and sustainability.

Keywords: quantitative models, sustainable development, urban form, Sanandaj City.
\end{abstract}

\section{INTRODUCTION}

The main purpose of this study is to identify and evaluate the level of sustainability for physical urban form in the Sanandaj City, located on the in western side of Iran. This city has experienced dramatic changes in size and shape during last 30 years. A greater understanding of the sustainable urban form that contributes to the quality of a community is needed by design and planning professionals to be able to play a more influential role in the preservation, enhancement and development of successful community environment especially for Iranian cities.

The rapid urbanization in Iran in the second half of the 20th century has mostly manifested itself in the emergence of large cities without specific elements of Iranian culture. This will isolate them from their rich architectural and urban design heritage. In addition, the old city centers, which are the only remnant of the glorious past, are slowly vanishing, due to modern development.

While sustainability of city is multifaceted, physical urban form and its relation to the efficiency of the city is the most acknowledged issue in the world environmental agenda. Many scholars argue that sustainable urban form is a key towards achieving a sustainable development and that quality of life is one of its vital components. Moreover, as cities promote the goal of becoming livable through compact polycentric approaches; potential importance to spatial factors in determining well-being grows more vital. Yet there exists very little empirical research to establish this claim. Hence urban forms cannot be considered 'sustainable' in the full sense if they are not acceptable to people as places to live, work and interact.

Today it is fully accepted that to achieve sustainable urban form, particularly in relation to land functionality and planning policy, the most efficient solution must be performed using compact city model. This model consists of a city shape with high density and mixed land use. Therefore, an understanding of the physical development pattern in order to navigate it towards sustainable urban development is essential. A vital element for this acceptance is the measurement of compactness/sprawl. Hence this 
study aims to apply quantitative analysis of urban form to verify whether the current progression of case study is really sustainable or not?

\section{URBANIZATION AND CHALLENGES IN IRAN}

Since the early 20th century, most cities in Iran have experienced dramatic increase in structure, which transformed the city area into diverse subareas due to intra-regional spatial differentiation. The traditional dichotomous urban structure of the city and the suburbs are not well suited for intraregional analyses and policy implications. Based on the population growth rate and the current urbanization trend, it projected that the population will be 130 million [1].

In order to meet the demand of this increasing population, a lot of land will be consumed. The rapid urbanization in Iran in the second half of the 20th century has mostly manifested itself in the emergence of large cities without specific elements of Iranian culture. This will isolate them from their rich architectural and urban design heritage. In addition, the old city centers that are the only witness of the glorious past are slowly vanishing, due by modern development.

Until few decades ago there was a balance between urban development and population growth, but recently, there are some problems. With new development, cities especially big ones, changed rapidly. These changes are swiftly extending the boundaries with unbalanced figures. Indeed, development of these kinds led to a lack of proportion between land use and per capita of the population.

Recent social and economic reforms in Iran influenced growth and urban physical development, leading to the growth of the services industry. This in turn has led to an increase in the population of industrial areas of the cities, since a lot of people migrated from rural area and converted smaller cities to larger cities [2].

Three important factors are related to the growth of Iranian city. These are natural population growth, migration from rural areas to cities and transformation of settlements from rural to urban state [3]. The most significant challenges for Iranian cities are indiscriminate expansion of metropolitan area, persistent migration to urban areas, poor distribution of population on land and informal settlements, land scarcity, expensive housing, traffic-related problems and pollution [4].

The reason for the imbalance in metropolitan areas in Iran has different dimensions, which include conflict between capacity of natural environment and the percentage of population. For instance, imbalance between functions of the central cities with the regions or imbalance in location of metropolitan areas when compared with the whole urban networks in the regions and imbalance between rural and urbanization systems in metropolitan areas. And finally a mix of urban elements that are incompatible with each other. The conflict between urban texture and inner city structure is also worth considering [5].

The problem of rapid growth and expansion of the city landscape in Iran is perhaps more acute than other developed and developing countries. This problem has become more complex after the Islamic Revolution. The revolution was followed by a long war (1980-1988) with Iraq, which halted economic development. In this period, planning for national development was subordinate to planning for the war. The era was characterized by rapid population growth, weak economic performance, a sharp decline in per capita income with a resultant fall in living standards for the average Iranian household [6].

The reasons and causes of this phenomenon in Iran can be classified as follows [7]:

- The lack of planning in the use of land has disarrayed the country's urban system. The growth rates of urban population, urban hierarchy and the number of cities are not compliant with the planning standards. The position and capacity of the cities have not been explained in the economic and social development of the country.

- The economic and social developments have not taken into consideration the position and capacity of the cities. 
- The transfer of land policy and land preparation also contributes to the physical expansion of cities.

- Lack of a comprehensive approach to the new form and construction of the city.

The population and housing results from 2008 [8] shows that nearly two-thirds of total population live in urban area and the trend for the next decade shows the same continuous increase in urban population. It also indicates that much of the population is concentrated in few metropolises. About $27 \%$ of the immigrants from 1996 to 2006 have been absorbed by six metropolitan areas. In 1976, one city had a population more than one million. The rate of urbanization between 1986 and 1996 was 54.3 and the number of cities that had a population more than one million increased to four. The majority of population in 1996 concentrated in five big cities, namely, Tehran, Mashhad, Esfahan, Tabriz and Shiraz. According to the last survey in Iran, there are six metropolitan areas with population more than one million each. This shows increase in number of the cities and population concentration around metropolitan areas will continue to increase in the future. Figure 1 shows the rate of urbanization in Iran within 50 years [8].

Inconsistency and cluttering are the factors in Iranian urban areas that prevented the metropolitan area from having standard values. Unsuitability of land uses, bizarre perspective and poor landscape have resulted in mushroom-like cities. Rapid urban development without planning in terms of interconnectivity is another issue. The lack of balance in many fields has also contributed to the crisis. Unsustainable structures of the big cities in Iran relate to inaccurate policies, imprecise planning in the development of the metropolitan areas and inappropriate regulations. There is lack of hierarchy for distribution of population in various level of the country and lack of hierarchy in terms of urban planning as a whole.

\section{URBAN PHYSICAL DEVELOPMENT IN SANANDAJ CITY}

The issue of sustainable urban form is becoming ever more important, taking into consideration the contemporary urban population and physical growth throughout the world and the concern for sustainable development especially for Iranian cities. The aim of this section is the description of the contemporary urban structure of the city from a sustainability perspective. This will be accomplished through the critical review of past and present planning priorities relating to sustainability, sprawl and compactness and through an assessment by quantitative techniques of urban sprawl or compactness in Sanandaj City.

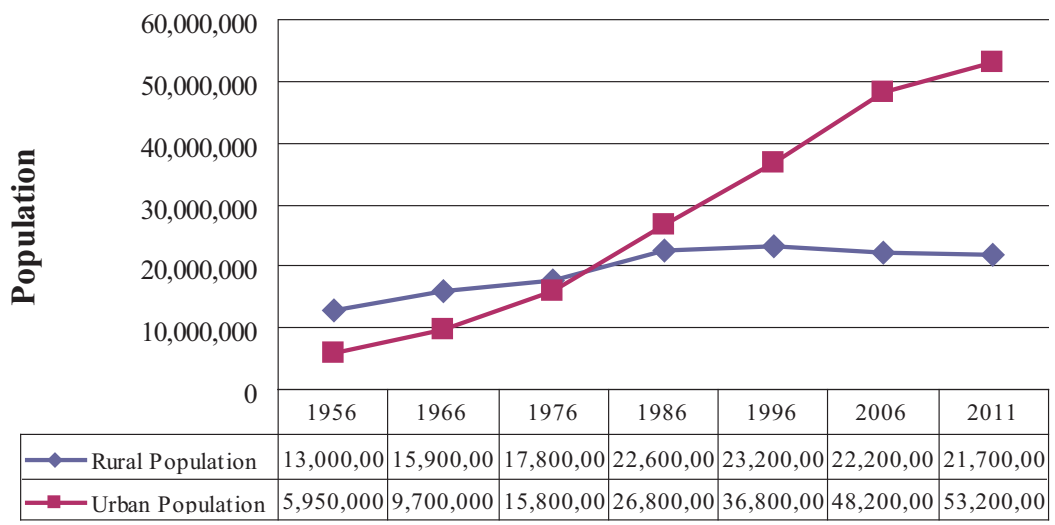

Figure 1: Population distribution by urban and rural area in Iran from 1956 to 2006. Source: [8] 
Sanandaj, center of Kurdistan Province, in terms of geographic location is located on 35 degrees 20 minutes north latitude and 47 degrees 18 minutes east longitude of Greenwich meridian and 15 degrees west longitude of Tehran meridian. Considering the geographic situation, it is located in the west side of Iran.

Topography of the city and the mountains surrounding it naturally confines the city in spatial bowl and the main and subcommunication networks. Sanandaj's spatial development was significantly affected by the imposed war, and also by the rural to urban immigration. A series of factors such as centrality, increased unemployment, unbalanced growth and physical development of the city, marginalization and arbitrary banning of resources which has filled the lives of present and future generations with deficiencies.

After the Islamic Revolution in 1979, urbanization of the country entered into a new phase of rapid growth. So that on one side the city faced transformation of concepts and functions and on the other side chaos and lack of limitations. These issues together with Iraq's war against Iran and regional conflicts led to excessive migration from villages and cities in the vicinity to Sanandaj City. So in this period Sanandaj faced its maximum physical growth.

Sanandaj City as the political-administrative center of Kurdistan Province and metropolis of the region has the dominant population. It is the economic and political hub of the province and due to its particular structure; it presents various services and facilities. It is a look up location for villagers, residents of other cities and even cities outside the province as well, so that probably Ehlers' term [9], 'exclusive usurpation of supplying the needs' can be attributed to it.

Accordingly, the city's growth was limited over a period of two hundred years. From 1951 onwards and for the last 40 years the city's population has increased 11 times. According to the latest statistics from the management and planning organization of Kurdistan, the city population was estimated at 316,832 in 2006 [8].

Demographic changes of recent years in Sanandaj City show that maximum urban growth in Sanandaj City has been between the years 1976 and1986. This decade is a period of social and political crisis in Iran, particularly in Kurdistan. Outcomes after the implementation of land reforms, the Islamic Revolution and the resulting unrest and imposed war provided a suitable ground for migration to urban areas, land utilization by speculators to capture the public lands and pastures surrounding the city.

Therefore, during the past three decades this city had a rapid increase in population and the expansion of the surface. Also in this period, the area (or the legal border of the city) has increased from 397 hectare to 3,815 hectare, but the population density has decreased from 241.5 in 1976 to 83 in 2006. This reflects the characteristics of urban creep and sprawl. In the 30-year period from 1976 to 2006, Sanandaj's area is nearly 10 times larger.

The city's physical growth during this period was 3,418 hectares and annually on an average has caused changing the functionality of 114 hectares of arable land, gardens and pastures into urban construction. During this period, the physical expansion of Sanandaj City has changed surrounding areas (rural, arable land, gardens and pastures) greatly. So that in this period, eight villages with arable land, gardens and meadows have become embedded because of city creep and sprawl and no longer have any sign of their rural structure.

During recent decades, the demographic and physical features of Sanandaj have disproportionately changed. Because of rapid development, the city has become characterized by juxtaposition of building typologies and urban forms. The city may be said to be losing identity as large sections of traditional urban fabric are being replaced. This form of growth negatively affects the use of resources and environment. Table 1 shows the 30 years' average growth rate in both population and urban area during the last 30 years. 
Table 1: Changes in population and area of the city of Sanandaj City during 1976-2006.

\begin{tabular}{|c|c|c|c|c|c|}
\hline \multirow[t]{4}{*}{ Year } & \multicolumn{2}{|c|}{ Demographic Changes } & \multicolumn{3}{|c|}{ Area Changes } \\
\hline & & The Average & & The Average & Changes in \\
\hline & & Annual Growth & & Annual Growth & Population \\
\hline & Total Population & Rate & Area (Hectares) & Rate & Density \\
\hline 1976 & 95,872 & - & 397 & - & 241.5 \\
\hline 1986 & 204,537 & 7.87 & 1,703 & 15.67 & 120.1 \\
\hline 1996 & 277,808 & 3.11 & 3,500 & 7.47 & 79.4 \\
\hline 2006 & 316,832 & 1.32 & 3,815 & 0.86 & 83.404 \\
\hline \multicolumn{2}{|c|}{ 30-Year average growth rate } & 4.06 & - & 7.83 & - \\
\hline
\end{tabular}

Source: Processing based on statistical yearbook of Kurdistan Province, 1976-2006

and organization of housing and urban development in Kurdistan Province, 2008.

This rapid increase in population, followed by immigration from rural to urban areas, natural growth (birth rate) and non-compliance with the population growth in most areas of the city's infrastructure capacity have been questioned in terms of sustainable development. Consequently, residents are using the land around the city disproportionately, thereby causing a lack of harmonious growth in the city.

According to master plan of the city, population density in region scale is volatility, while the gross population density in total area of the city is relatively favorable. This amount in region 2 and 3 is low and in region 1 is very high. The level of heterogeneity in population density in Sanandaj City ultimately led to the poor access to urban services and facilities.

In terms of physical space, the per capita in urban areas is not appropriate. Green and recreation spaces in the city with a per capita of about 5.08 meter (7-12 meter of Iran city's common per capita), educational spaces, 6.99 square meter (3-5 square meter of Iran city's common per capita), cultural spaces 0.55 square meter (5.1-0.75 square meter of Iran city's common per capita), sport spaces 0.61 (2-2.5 meter of Iran city's common per capita).Treatment facilities, including, health spaces of 0.02 square meter (from 1.5 to 0.75 square meter of Iran city's common per capita) as well as traffic congestion with traffic tie-ups in the streets and main passages are other issues raised in this regard.

\section{APPLYING QUANTITATIVE TECHNIQUES FOR MEASURING SUSTAINABLE URBAN FORM IN SANANDAJ CITY}

'Horizontal expansion of the city' is a term that has been added to the planning research literature in the form of 'sprawl' in the last 50 years. The use of the term dates back to the mid-20th century when the arbitrary banning of personal automobile, expansion of highway system and also urban spaces expansion in America flourished. Urban sprawl has different dimensions in which low values of each of these dimensions can be more diffused [10].

Ewing [11] argues that urban sprawl is defined by scattered development, development of large areas of low densities and development of commercial strips. Other researchers like Burchell (1998) added that sprawl includes both residential and non-residential development and that different land uses often are segregated from each other [12].

The compact city can be considered as the opposite of urban sprawl [13]. As one of the multitude of different models for sustainable urban development, the compact city approach is widely 


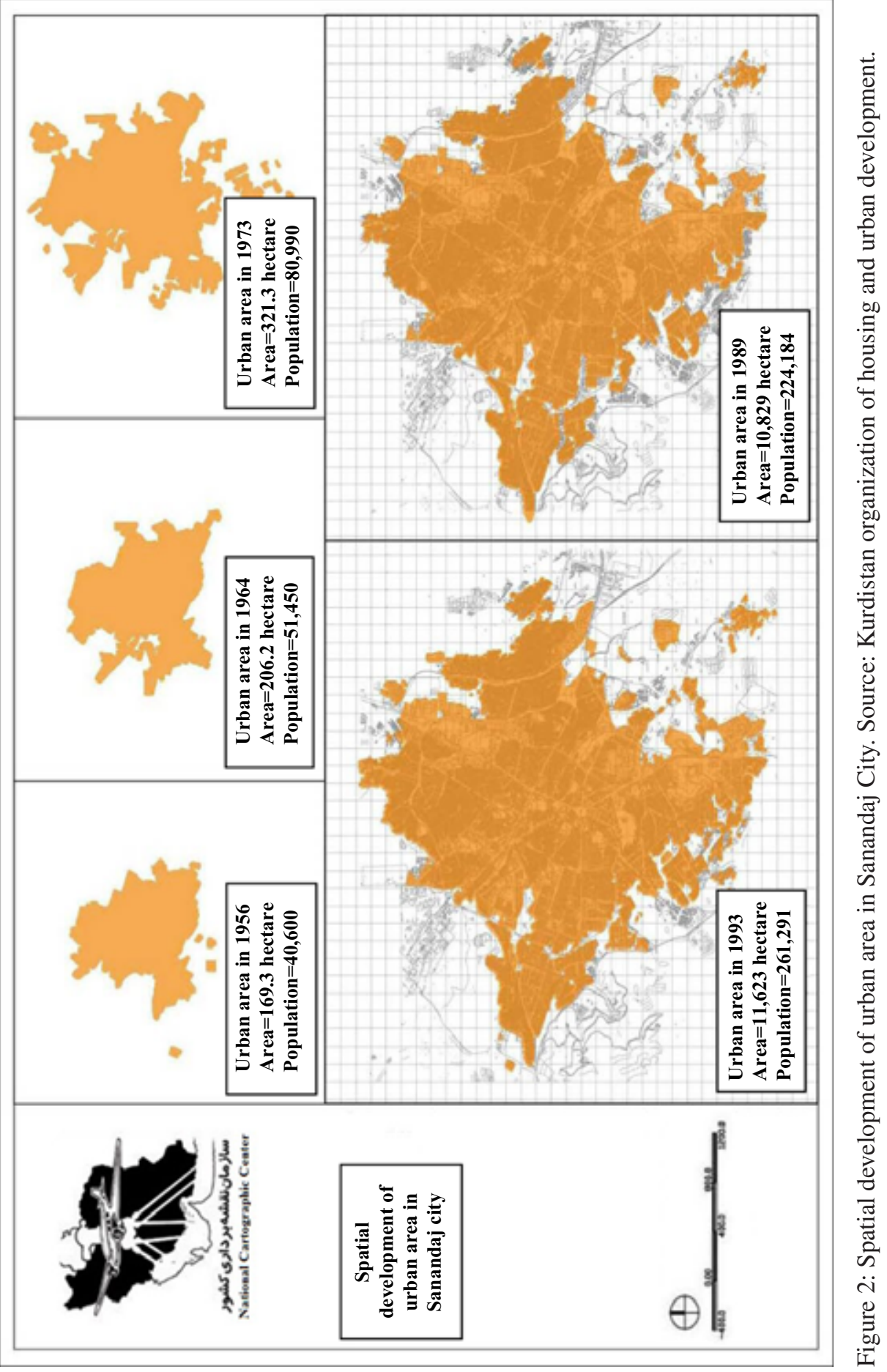


recognized as key to achieve a sustainable urban form [14]. The compact city idea implies advocacy of high densities and mixed land uses, and has been said to provide protection for the countryside and lower emissions of greenhouse gas through reduction in vehicle use [15]. Furthermore, the compact city is believed to improve the economic potential in neighborhoods, provide higher levels of culture and social diversity as well as the safety and social equitability.

There are different models to analyze the shape of the physical expansion of cities and the way they are planned. Some of the researchers have pointed obvious differences in the shape of a city using indexes such as density, diffraction, scattering, and availability (e.g. [10, 16-19]).

Recently, quantitative methods have been used as tools for systematic classification and analysis of patterns of urban growth. In this study, two main techniques to evaluate the size and shape of urban forms were used. These are the Shannon entropy and Holdren apportioning models, which are discussed in the following sections.

\subsection{The Shannon entropy}

The technique is used for analyzing the unpleasant urban growth and the level of sprawl in urban area. It is also used for the analysis and determination of chaos phenomenon of urban growth and to analyze the shape and size of the city. Another use is to find a suitable technique to plan for future physical development. Shannon's entropy used to measure the degree of spatial concentration and dispersion exhibited by geographical variable [20].

Although several technique such as the Shannon entropy, Holdren, Moran and Gray coefficient measure the shape of the city, potentially Gary and Moran coefficient defines dispersion from centrality by focusing on spatial structure, but their interpretation is often complex [21].

However, the relative entropy index (an index derived from the Shannon entropy to convert values to a range between zero and one) is better than others, because it is not affected by the number of subregions [22]. The index also can be used to analyze the data and the degree of organization of a system [23].

The overall structure is as follows [24]:

$$
H=-\sum_{i=1}^{N} P i \times \operatorname{Ln}(P i)
$$

In which:

$H=$ the Shannon entropy value

$P i=$ the proportion of built area (overall residential density) zone $\mathrm{i}$ to the total built areas

$N=$ total area

The value of Shannon entropy is between zeros and $L n(n)$, in which zero indicates that the physical development is too dense (compact), while the $\operatorname{Ln}(n)$ represents the physical development is scattered throughout the city. In fact, when the entropy value is larger than $\operatorname{Ln}(n)$, ugly urban growth (sprawl) has occurred.

In 1991, total built area of Sanandaj City was 1,448 hectares and share of each of the three regions was 587, 716 and 145 hectares, respectively. Entropy value for the city of Sanandaj in 1991 was 0.9443 and the estimated maximum value of $\operatorname{Ln}(3)=2.23016$. Large difference between the maximum entropy values indicates that the physical growth of the city has been dense and continuous.

The entropy value in 2006 was equal to 2.92, which represents a scattered and disorganized physical development and shows the tendency towards imbalance in physical development of Sanandaj City. 
Table 2: Calculate the entropy values in urban areas.

\begin{tabular}{|c|c|c|c|c|}
\hline District & Built-up Area (Hectare) & $P i$ & $\operatorname{Ln}(P i)$ & $P i \times \operatorname{Ln}(P i)$ \\
\hline 1 & 41.8617 & 0.0195 & -3.9345 & -0.0769 \\
\hline 2 & 55.3721 & 0.0258 & -3.6548 & -0.0945 \\
\hline 3 & 88.6747 & 0.0414 & -3.1839 & -0.1318 \\
\hline 4 & 189.9950 & 0.0887 & -2.4219 & -0.2149 \\
\hline $4 \mathrm{~b}$ & 73.0984 & 0.0341 & -3.3771 & -0.1153 \\
\hline 5 & 108.3666 & 0.0506 & -2.9834 & -0.1510 \\
\hline 6 & 135.9814 & 0.0635 & -2.7564 & -0.1750 \\
\hline 7 & 19.8265 & 0.0092 & -4.6819 & -0.0433 \\
\hline 8 & 142.7112 & 0.0666 & -2.7081 & -0.1805 \\
\hline 9 & 148.3154 & 0.0692 & -2.6695 & -0.1849 \\
\hline 10 & 120.2958 & 0.0561 & -2.8789 & -0.1617 \\
\hline 11 & 44.5397 & 0.0208 & -3.8725 & -0.0805 \\
\hline 12 & 143.7920 & 0.0671 & -2.7005 & -0.1813 \\
\hline 13 & 109.9869 & 0.0513 & -2.9685 & -0.1525 \\
\hline 14 & 101.5710 & 0.0474 & -3.0481 & -0.1446 \\
\hline $14 \mathrm{~b}$ & 30.7890 & 0.0143 & -4.2417 & -0.0610 \\
\hline 15 & 115.3992 & 0.0539 & -2.9205 & -0.1574 \\
\hline 16 & 60.6939 & 0.0283 & -3.5630 & -0.1010 \\
\hline 17 & 184.3977 & 0.0861 & -2.4518 & -0.2111 \\
\hline 18 & 86.8224 & 0.0405 & -3.2050 & -0.1299 \\
\hline 19 & 138.2971 & 0.06460 & -2.73952 & -0.1769 \\
\hline Total & 2140.7873 & $\sum P i=1$ & & -2.9270 \\
\hline
\end{tabular}

Being close to the maximum entropy value of $\operatorname{Ln}(21)=3.04$, reflects the scattered growth and physical development of the city. Calculations show that the physical expansion of the city during the last 10 years is even more diffused. Table 2 shows the calculation.

\subsection{The Holdren apportioning method}

A method for quantifying the respective contributions of population growth and changes in consumption per capita of any type of resource was discussed in a landmark 1991 paper by Harvard physicist Prof. John Holdren. This method can be applied to many types of population/resource consumption analysis. By this method we can determine the level of sharing of sprawl due to population growth and land area per capita.

In this method, he used the gross per capita formula for which the equation is as follows [24]:

$$
a=\frac{A}{P}
$$

In eqn (1), area of urbanized land for the average resident $(a)$ is equivalent to dividing the area of total urbanized land in a city and its suburbs $(A)$ to the population $(P)$. According to eqn $(1)$, total land area occupied by the city $(A)$ is equal to per capita land use $(a)$ and population $(p)$. Then we have 


$$
A=P \times a
$$

According to the Holdren model, if on a given period $(\Delta t)$, the population grows with a growth $(\Delta p)$ and the per capita land is changed with the equal $(\Delta p)$, total area increases by $(\Delta A)$, using eqn (2), the whole metropolitan area is obtained:

$$
A+\Delta A=(P+\Delta P) \times(a \times \Delta a)
$$

Subtracting eqn (2) from eqn (3) and dividing through by $(A)$, we can obtain the changes of $(\triangle A / A)$ area, which is converted into the city during the period $(\Delta t)$.

According to the Holdren model, eqn (4) states that a growth and expansion percentage of a city is equal to the sum of population growth and gross per capita growth. The relation (4) is

$$
\frac{\Delta A}{A}=\frac{\Delta P}{P}+\frac{\Delta a}{a}+\left(\frac{\Delta P}{P}\right) \times\left(\frac{\Delta a}{a}\right)
$$

Overall percentage land area growth $=$ overall percentage population growth + overall percentage per capita growth

Accordingly, the share of population growth of the land (sprawl) is obtained by the ratio of percentage change of total population in a period to percentage change of total land area in the same period which can be stated as follows:

Growth share of the earth $=($ growth percentage of the total population $) /$ (total percentage of land area)

The per capita share of land can be calculated in the same way as follows:

$$
\text { Per capita: land use share growth }=\frac{\text { Overal percentage per capita land use growth }}{\text { Overall percentage land area growth }}
$$

Based on population growth model, Holdren presents a general growth model to complete his model

$$
P_{(T)}=P_{0}\left(1+g_{p}\right)^{t}
$$

wherein $P_{(T)}$ is the population at time $t, P_{0}$ is the primary population, the population growth rate during the time interval. To solve $g_{p}$ the following equation can be used:

$$
\operatorname{Ln}\left(1+g_{p}\right)=\left(\frac{1}{t}\right) \operatorname{Ln}\left(\frac{P_{t}}{P_{0}}\right)
$$

Because $\operatorname{Ln}(1+x)$ for values lower than $x$ are almost near $x$, eqn (9) can be written as follows:

$$
g_{p}=\left(\frac{1}{t}\right) \operatorname{Ln}\left(\frac{P_{t}}{p_{0}}\right)
$$

The infringing of growth rate for land area $(A)$ and land functionality $(a)$ can also be written as follows:

$$
\begin{aligned}
& g_{A}=\left(\frac{1}{t}\right) \operatorname{Ln}\left(\frac{A_{t}}{A_{0}}\right) \\
& g_{a}=\left(\frac{1}{t}\right) \operatorname{Ln}\left(\frac{a_{t}}{a_{0}}\right)
\end{aligned}
$$


So, according to these three population growth rates, the Holdren equation can be written as follows:

$$
g_{p}=g_{a}=g_{A}
$$

$L n$ (final population/initial population) + Ln (final per capita land area/initial per capita land area) $=\mathrm{Ln}$ (final total land area/initial total land area).

By substituting the equation results obtained from the Holdren model we can obtain the following:

$$
\begin{gathered}
\operatorname{Ln}\left(\frac{316,832}{95,872}\right)+\operatorname{Ln}\left(\frac{0.0120}{0.0041}\right)=\operatorname{Ln}\left(\frac{3,815}{397}\right) \\
\operatorname{Ln}(3.304)+\operatorname{Ln}(2.898)=\operatorname{Ln}(9.609) \\
1.1953+1.06419=2.262 \\
\left(\frac{1.1953}{2.262}\right)+\left(\frac{1.06419}{2.262}\right)=1 \\
0.53+0.47=1
\end{gathered}
$$

In the case of Sanandaj, the urbanized area from 1976 to 2006, the share of sprawl due to population growth was $53 \%$ [100\% $\times(1.1953 / 2.262)]$, while declining density (i.e. an increase in land area per capita) accounted for $47 \%$ [100\% $\times(1.06419 / 2.262)]$.

This procedure leads to many urban problems. Despite high volume of recycled land in the city, the city managers lack clear plans for the expansion of the city. Therefore, the need for land is fulfilled through physical expansion of the city and not through recycling the land through inner structures.

Sanandaj City, as the capital of Kurdistan Province and as one of the biggest cities in the west of Iran, is experiencing such transformation in two fields: the first can be seen in the way in which the city has started to expand and the second is problems that are evident at the level of its different consistencies. The particular characteristics of Sanandaj have predominantly given a new shape to its socio-spatial organization. In recent decades, the city has experienced a rapid urban growth. One aspect of this phenomenon is seen in its urban fragmentation which was based on different urban environments. This has given rise to a situation where Sanandaj City has become an expanded city without sufficient urban infrastructure.

The social areas that have appeared in this city constitute separate socio-spatial sectors which have developed with particular socio-physical characteristics. The current modulating urban network is not able to support sufficiently the links between these sectors.

\section{CONCLUSION}

Urban sprawl has increasingly become a major issue in the global trend towards urbanization, and is faced not only by developed countries but also by developing countries. Large urban centers and medium and small cities alike are also facing the same problem. Urban sprawl raises social and environmental concerns at the same time that shows a multiplicity of divergent trajectories that somehow defy the dominance of homogenous characteristics around the world. Urban sprawl has many different characteristics. It affects the environment, economy and society in multiple ways. When adding sustainability to urban form, often an impression is produced of a more compact development pattern. This paper serves only to illustrate how some assessments can be made. The medium size of Sanandaj City does not need quantitative assessment required for larger settlements. But the 
analytic role is of vital importance as support for important policy decisions relating to urban development.

As every city's model of physical development has a great influence on the sustainability of its growth, urban managers and planners should have enough information on the existent models of urban physical and spatial expansion in order to design the model for urban sustainable development. In the present study, in order to investigate and analyze Sanandaj City's model of physical growth, the models of Holdern and Shannon's Entropy were employed.

Findings using the Holdern model indicate that only 53\% of urban growth has been the result of actual urban growth. About $47 \%$ of urban growth is related to urban sprawl and horizontal growth, which contribute to the gross density of population and the increase in urban gross land per capita. Also, the value of Shannon's Entropy is 2.92 for 2006 whereas the maximum value is $\operatorname{Ln}(21)=3.04$. The closeness of the entropy value to maximum values shows the sprawl growth of urban physical development.

On the basis of the methods used, it can be concluded that the physical development pattern of Sanandaj City during the last decade tends to tilt towards urban sprawl. According to the findings of the researches on unsustainable pattern of urban growth, many negative consequences in different economic, social and environmental sectors are created. This includes loss of agricultural land surrounding the city, degradation and pollution of soil, water sources and air pollution. There is also increase in length and travel time to urban areas.

Most importantly, Research and Professional Committee of Urban Planning in Iran is faced with three fundamental issues in terms of sustainable urban form [25]:

- Lack of attention to qualitative aspects of shape of the city in support of human psychological demands of space along with the functional, economical, legal and socio-political expectations. The effect of civilian traffic is also not given much attention.

- Neglecting the formation of features in traditional urban planning in Iran.

- Lack of a comprehensive approach to the new form of the city, new construction and fast and growing expansion.

There are two concerns behind the issue of urban form in Sanandaj City. The first concern is the dramatic urban changes that have been witnessed in the contemporary period in this city (particularly in the years after the revolution in Iran in 1979). These urban transformations were the major source of the emergence of many differences at the level of its residential areas. This is the issue that the practice of urban ecology proposes as a general process for contemporary cities.

The second concern was the lack of understanding and sensitivity among urban authorities to the definition of sustainable urban form and understanding relationship between the city, residents and physical form. In this sense, the lack of sufficient understanding of local variables has been criticized through the ideas of sustainability in Sanandaj City. Based on this argument, policy for the development of urban areas should consider the reality of physical form not just in macro sociological points of view. Significantly, it should take into account the facts of urban form, which are more emphasized in the frame of reference of recent micro analysis.

The provided tools and techniques were appropriate for measuring the degree of dispersion and compactness. It is a partial response to the concerns of urban policy makers in the present situation and future trends of the city shape that are present in Iran's urban planning literature. It is hoped that this research is the beginning of a study of such functional issues in this field. Hence, the need for change in attitudes of urban planning and management and using the solution for more compression is felt. 
In order to achieve compact growth and avoid the sprawl growth of Sanandaj City, the decentralized centralization (changing one polar city into multicenter city based on compact construction and multiplication of activities in selected subcenters) should be followed. Emphasis should be placed on urban smart growth strategies and principles as a model for future urban development. To achieve this model, the following actions are recommended:

- Centralized local activities in local communities and as a result, elevating life quality, security and dynamism as well as supporting occupations and services, leading to the creation of environment for flourishing economic and commercial activities.

- Making use of aggregation and compact models in new constructions in order to avoid urban sprawl growth.

- Encouraging citizens to participate in the decision regarding development.

- Promoting infill development.

\section{REFERENCES}

[1] Habbibi, K. \& Poorahmad, A., Analysis of Spatial Development of Sanandaj City by Geographic Information System, Vol. 1, Kurdistan University: Iran, 2005.

[2] Mashhadizadeh, v., An Analysis of Urban Planning Characteristics in Iran, Iran University of Science \& Technology: Iran, 1996.

[3] Charehjoo F. \& Chin Siong, H., The need for smart growth concept for Iranian cities, presented at the 3rd International Conference on Engineering Science and Humanities, Universiti Teknologi Malaysia, 2010.

[4] Izadi, H., The trend of changing rural area to urban area in Iran, challenges and advantages, presented at the Iranian Urbanization Issues Conference, 2003.

[5] Saidnia, A., The structure of unsustainable metropolitan Tehran, presented at the Iranian Urbanization Issues Conference, 2003.

[6] Atash, F., New Towns and Future Urbanization in Iran. TWPR, 22, p. 48, 2000.

[7] Azizi, M.M., Density in Urban Planning, Principles and Criteria for Density Determination, University of Tehran: Iran, 2003.

[8] SCI, General Population and Housing Census Results: Statistical Center of Iran., 2008.

[9] Ehlers, E., Capitalism and Urban Genesis in The Islamic East ( The Case of Iran), Translate by Abbas Saidi. : Tehran, Monshi, 2001.

[10] Galster, G., Hanson, R., Ratcliffe, M.R., Wolman, H., Coleman, S. \& Freihage, J., Wrestling sprawl to the ground: Defining and measuring an elusive concept. Housing Policy Debate, 12, pp. 681-717, 2001. doi: http://dx.doi.org/10.1080/10511482.2001.9521426

[11] Ewing, R., Is Los Angeles-Style sprawl desirable. American Planning Association, 63, pp. 107-126, 1997. doi: http://dx.doi.org/10.1080/01944369708975728

[12] Burchell, R.W., Shad, N.A., Listokin, A. \& Philips, H., The Costs of Sprawl Revisited. Washington D.C.: National Academy Press, 1998.

[13] Neuman, M. The compact city fallacy. Planning Education and Research, 25, pp. 11-26, 2005. doi: http://dx.doi.org/10.1177/0739456X04270466

[14] Burton, E. The Potential of the Compact City for Promoting Social Equity. Ur: Williams, K., Burton, E. \& Jenks, M. (eds.). E\& FN Spon: New York, 2000.

[15] Jenks, M., Burton, E. \& Williams, K., The Compact City. E\& FN Spon: New York, 1996.

[16] Huang, J., Lu, X.X. \& Sellers, J.M. A global comparative analysis of urban form: Applying spatial metrics and remote sensing. Landscape and urban planning, 82, pp. 184-197, 2007. doi: http://dx.doi.org/10.1016/j.landurbplan.2007.02.010 
[17] Tsai, Y. Quantifying urban form: Compactness versus 'sprawl'. Urban Studies, 42, pp. 141-161, 2005. doi: http://dx.doi.org/10.1080/0042098042000309748

[18] Ewing, R., Pendall, R. \& Chen, D., Measuring Sprawl and Its Impact, Smart Growth America: USA, 2002.

[19] Rahnama, M.R. \& Abaszadeh, G., A Comparative study and analyzing compactness/ sprawl ratio in the metropolitan cities of Mashhad and Sydney. Geography and Regional Development, 6, pp. 101-128, 2006.

[20] Thomas, R.W., Information Statistics in Geography, Norwich: W. H Hutchins \& Sons, 1981.

[21] Anselin, L., Space Stat Version 1.80: Users' Guide, Regional Research Institute, West Virginia University: Morgantown, WV, USA, 1995.

[22] Rahnama M.R. \& Abaszadeh, G., Principles and Models for Measuring Physical Form of Cities, Mashhad University: Iran, 2008.

[23] Malek hoseini, A., Analyzing the spatial organization of the Markazi Province. Amayesh, 1, p. 1, 2006.

[24] Hekmatnia H. \& Moosavi, M., The Application of Model in Geography, Focusing on Urban and Regional Planning. Elm e Navin e Yazd Publication: Yazd 2006.

[25] Habib, F. To explore the meaning of urban form. Honar-ha-ye Ziba, 25, p. 32, 2006. 